\title{
A tool for evaluation of lifecycle cost of water production for small-scale community projects
}

\author{
Shivendra Kumar ${ }^{\mathrm{a}}$, Andrew Groth ${ }^{\mathrm{b}}$ and Ljubo Vlacic ${ }^{\mathrm{b}}$ \\ ${ }^{a}$ Corresponding author. Evoqua Water Technologies, 15 Blackman Crescent, South Windsor, New South Wales 2756, \\ Australia.E-mail: shivendra.kumar@evoqua.com \\ ${ }^{b}$ School of Engineering, Griffith University, 170 Kessels Road, Nathan, Queensland 4111, Australia
}

\begin{abstract}
The aim of this study is to develop an analytical tool to estimate lifecycle cost for water service provision for small-scale community water projects. The primary objective is to provide an analytical estimation tool to governments, funding agencies and communities to ensure sustainability of water projects. The secondary objective is to use the tool as an industry benchmarking aid to empower governments, funding agencies and technology developers to support technologies that would further lower the cost of water production. The tool firstly considers capital expenditure required for a given water service system, focusing on equipment manufacturing costs in detail. Thereafter, it considers the operational expenditure associated with ongoing functioning of the water treatment plant. The developed tool is validated using five simulation scenarios. Results shows that the tool can be used to evaluate the performance of a water service system even if the water services systems are operated using varying strategies. The developed tool also enables effective decision making by testing alternatives and addressing the water service system lifecycle aspects and, thus, making the cost of water production manageable. This paper also introduces the web version of the tool, which is available to communities, governments, technology developers and funding agencies to use.
\end{abstract}

Keywords: Lifecycle cost modelling; Water production cost

\section{Nomenclature}

$\mathrm{Bl} \quad$ Cost of building

C Cost of chemicals

CAPEX Capital expenditure

E Energy costs

$E q \quad$ Cost of equipment

$F \quad$ Facility management costs

doi: 10.2166/wp.2015.135

(C) IWA Publishing 2015 


$\begin{array}{ll}L & \text { Labour costs } \\ L a & \text { Cost of land } \\ M & \text { Maintenance and spare part costs } \\ \text { MLD } & \text { Mega litres per day } \\ \text { NGO } & \text { Non-governmental organization } \\ \text { OPEX } & \text { Operating expenditure } \\ P M & \text { Cost of project management } \\ t & \text { Estimated useful life of equipment } \\ \text { UV } & \text { Ultraviolet } \\ V_{\text {exp }} & \text { Estimated annual water production volume } \\ \text { WASH } & \text { Water, Sanitation and Hygiene } \\ \varepsilon & \text { Annual inflation factor } \\ \sigma & \text { Plant-aging factor }\end{array}$

\section{Introduction}

High quality water is recognized as an essential requirement for life and is also recognized as a resource which is fast becoming scarce (Kumar et al., 2014). Population growth and the resulting need for water for agricultural and industrial sectors has made water a valuable resource. With increase in costs of establishing and operating water treatment operations, due to increases in cost of real estate, labour and energy, along with tighter water quality guidelines, there is increased pressure on the cost of water production. Costs associated with extracting, treating and distributing drinking water is one of the reasons why millions in small communities do not have access to reliable drinking water.

Research has focused on impact of water quality on public health, monitoring metrics, development of new low cost water treatment technologies and sustainability. Availability of safe drinking water is considered as one of the components of public health. Jacangelo et al. (2006) discussed the Safe Drinking Water Act and how it aids in protecting public health by ensuring water continues to be free of emerging micro-contaminants. Jacangelo et al. (2006) mentioned the presence of pharmaceutical personal care products and hormonally active agents from domestic and agricultural use respectively as new threats to water quality. While the impact of low levels of exposure to the pharmaceutical and hormonally active agents has not been well defined, potential health effects include disruption to the human endocrine system. Storage of water in unprotected storage vessels has been identified as a public health issue by Graham \& VanDerslice (2007). Graham \& VanDerslice (2007) reported a reduction in the quality of stored water when compared to tap water. Unreliable and inconsistent supply of water has been linked to increased need for storage which in turn results in higher levels of coliforms. Graham \& VanDerslice (2007) suggested making water available consistently as the best solution and adding more free chlorine in larger storage tanks if water supply cannot be improved. Water quality has been linked to health issues in refugee camps also. Cronin et al. (2008) mentioned that people developed health issues within 24 hours in instances where there was not adequate provision of water. Cronin et al. (2008) called for integrated approaches to dealing with water provision in acute emergencies. The integrated approach will see an agreed priority assigned to food, nutrition and health interventions such as water and sanitation, access to non-food items etc. Expanding further from refugee camps to communities, Rizak \& Hrudey (2008) identified patterns in the recurrence of waterborne diseases in community managed systems. Monitoring and reporting of data is a powerful way to initiate action. Bartram (2008) started discussion on internationally accepted indicators that will stimulate action to improving water access, sanitation and hygiene. Bartram (2008), using case studies and examples, argued that current benchmarks are not adequate in ensuring water provision is compared against 
health improvement; some communities are exceeding benchmarks whereas others are not. With the example of comparing a protected well $30 \mathrm{~m}$ from home to where water is available in the home, Bartram (2008) suggested that the water sector needs a sequence of benchmarks for potentially different routes to improvement. Furthermore, water provision need not be confined only to households but expanded to community centres such as schools, markets and hospitals. Other researchers have also looked at metrics. Sullivan et al. (2003) proposed the Water Poverty Index which measures water stress at community and individual level. The metric proposes a consolidated index of measures such as access to water, available water quantity and environmental factors. Hutton et al. (2007) reported the findings of a global cost-benefits analysis of a range of interventions focused on water, sanitation and hygiene (WASH). The authors report a $\$ 5$ benefit in a base case for every $\$ 1$ invested. Capital and operational expenditure in WASH activities saved time, improved productivity and generated health care cost savings for communities. Kayser et al. (2013) presented an historical overview of various monitoring indicators that have been used to guide policy formulation and drive civil society influence on services intervention.

Sustainability is an important topic for water provision in small communities and it is receiving increasing focus. From managing growing water scarcity, preventing contamination of distribution systems to implementing innovative sustainable solutions, the challenges facing the WASH sector cannot be underestimated (Moe \& Rheingans, 2006). The authors further alert the readers through their paper that the world is littered with failed water service projects and broken pumps, systems unused due to lack of energy sources and spare parts that are difficult to find. Taylor (2009) also reported, using data gathered by WaterAid, that $46 \%$ of water points are not working and approximate operational life of $25 \%$ of the installations is less than 2 years.

Despite the issues of sustainability, water treatment technology has continued to develop for industrial and municipal sectors. Technology developed for those sectors is also applicable to small communities. Nevertheless, researchers have been working to develop improved methods to simplify water treatment while improving water safety for smaller remote communities. Clasen et al. (2007) studied cost effectiveness of various existing drinking water provision solutions such as boreholes and communal stand post systems alongside chlorination, filtration and disinfection systems. Like Clasen et al. (2007), Murphy et al. (2010) carried out an evaluation of technologies. Biosand and ceramic filters were compared by Murphy et al. (2010) using parameters identified as critical by local non-governmental organizations (NGOs) and the authors. Brownell et al. (2008), having identified the need for improved water treatment solutions for remote communities, proposed a point of use ultraviolet (UV) based solution. Similarly Muhammad et al. (2010) developed a composite cartridge system for use in poor water quality scenarios.

The benefits of the shift of focus toward technologies that deliver the innovative solutions that can make safe drinking water easily available to all will be significant. There is a need to recognize that water provision will improve quality of life starting from sanitation and hygiene. Bartram (2008) correctly pointed out that good sanitation protects health. Clasen et al. (2007) mentioned the economic burden waterborne diseases, such as diarrhoea, cause. With improved safe water provision, significant direct costs affecting the health sector and indirect costs affecting education and productivity could be improved. Haller et al. (2007) and Hutton et al. (2007) offered a more simplified view that any investment in safe water provision will yield earnings in improved productivity and savings in healthcare costs. Rizak \& Hrudey (2008) went even further to state that drinking water suppliers need to develop 
robust systems that can withstand any factors that may risk drinking water safety throughout the lifecycle of the water service system.

Despite an abundance of literature indicating increased focus on water quality and its impact on public health, there are no measures to model and reduce the cost of water production in smaller communities. Low cost of water production translates to efficient use of funds set aside for public health initiatives as well as sustainability of implemented solutions. Furthermore, determination of water production costs for projects upfront will ensure solutions are implemented with consideration of cost and sustainability. It is expected that this will drive innovation of cost effective technologies to improve accessibility to safe drinking water. It will also empower small communities to purchase and implement water production systems themselves.

In this study, the components that make up water service systems for community based small projects are defined with the intention of illustrating the cost of water production. This research benefits the community through the provision of an easily accessible model that can help estimate and report lifetime performance of water services. This helps ensure that public health improvements focused on safe drinking water, remain affordable and economically sustainable.

This paper presents an analytical model developed as part of this study that elaborates on the manufacturing and implementation costs of water treatment systems in small communities. The model will explain costs associated with main components, auxiliary components, capital costs and operational costs over the lifecycle of the water treatment system. Also, this study has developed a web tool that can accurately predict lifecycle costs of water services systems. That tool will be presented in the latter sections. This paper also shares analysis of simulation scenarios evaluated using the developed tool.

\section{Method}

\section{Water services lifecycle cost model development}

This section focuses on development of a model to determine costs associated with producing clean drinking water for community based small projects. The model is based on the process boundary diagram shown by Figure 1.

The model looks at determining the cost of all components illustrated above. A high-level computation of cost inputs is done to determine the cost of the capital equipment. The cost component of establishing and operating a system is stated, followed by analysis of every factor contributing to the cost of water production. The unit of measure for all costs in this paper is USD, although it can equally be presented in any other monetary unit.

Costs directly related to the establishment and upgrade of equipment for transferring, filtering and storing water is classified as capital cost. Capital costs consist of the following components.

- Land $(L a)$ - The size of land required will be the footprint of the building plus items such as access, storage for outdoor process equipment, waste management facility, and space dedicated for regulatory compliance. Included in the land capital cost is the cost of developing the land to make it usable for a water service system. 


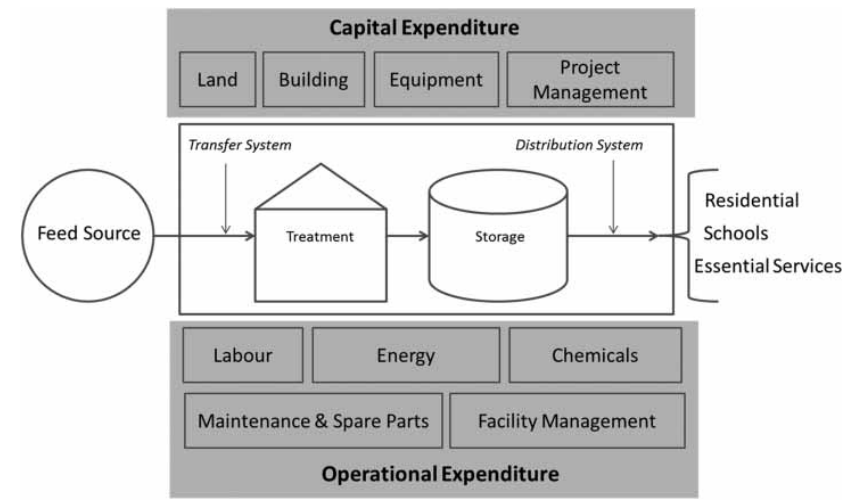

Fig. 1. Typical boundary diagram for community based small water projects.

- Building $(B l)$ - the building is required to sufficiently house equipment associated with the water service system. The size of the building will be directly proportional to the combined size of all equipment in the plant. Mid- to long-term expansion plans and regulatory requirements are also factored into determining the building size.

- Equipment $(E q)$ - cost to procure equipment for the water service system. It consists of equipment at the intake from the feed source to the distribution network.

- Project Management Costs $(P M)$ - costs to manage the project from start to finish ensuring compliance with safety and other regulatory requirements from governments and project sponsors.

Capital expenditure (CAPEX) is therefore calculated as follows:

$\operatorname{CAPEX}(\$)=\mathrm{Eq}+\mathrm{La}+\mathrm{Bl}+\mathrm{PM}^{\prime}$

$E q$ is one of the main sub-components of the CAPEX and is the sphere of influence where technology development and innovation will have the greatest cost improvement.

To operate water treatment and distribution systems, there are ongoing expenses. Unlike capital expenses that are one-time costs, operational expenses are recurring with time and water production. Operational expenditure typically includes:

- Maintenance and Spare Parts $(M)$ - to maintain and repair components in order to keep plant functioning at desired quality and efficiency levels.

- Energy $(E)$ - whether manual or harnessed, energy is required to keep the system operating. Pumps are usually the largest energy consumer in typical water treatment and distribution systems.

- Labour $(L)$ - to operate and maintain the equipment. This helps ensure availability, quality of water and long-term reliability of the equipment.

- Chemicals $(C)$ - depending on the system used and feed quality, chemicals maybe used to clean and sanitize treatment, transfer and storage systems.

- Facility management costs $(F)$ - although not significant, these costs are driven by the need to comply with regulatory standards, and maintain the facility, community education and protection of the feed source. 
Operational expenditure can vary, as it is dependent on economic factors such as inflation and government policies. Inflation would influence cost of energy and cost of chemicals and spares.

Operational expenditure can be stated as:

$\operatorname{OPEX}(\$)=\frac{\mathrm{M}+\mathrm{E}+\mathrm{L}+\mathrm{C}+\mathrm{F}}{\mathrm{V}_{\exp }}$

where $V_{\text {exp }}$ is the estimated volume of water production for 1 year.

The operational objectives of a community producing water will normally be tailored around equipment performance and reliability. However, more important than that is the cost of water production especially since it relates to sustainability. With the view of accomplishing performance and reliability objectives, the lifetime cost evaluation tool is presented. The tool measures the cost of water production in the units $\$ / \mathrm{m}^{3}$, although an equivalent $\$ / \mathrm{L}$ or other conversion units could be utilized.

The water production cost is therefore comprehensively stated as follows:

Water Production Cost $\left(\$ / \mathrm{m}^{3}\right)=\frac{(\mathrm{OPEX} \times \mathrm{V} \times \mathrm{t} \times \varepsilon \times \sigma)+\mathrm{CAPEX}}{\left(\mathrm{V}_{\exp } \times \mathrm{t} \times \sigma\right)}$

where $t$ is the useful life of the equipment, $\varepsilon$ is the annual inflation factor and $\sigma$ is the plant-aging factor.

Manufacturers or system developers will predict equipment life $t$ from statistical, theoretical, demonstrated or accelerated testing data. Demonstrated data can only be used for mature technologies or standard components. Depending on the technology and feed source, the efficiency of the system will be expected to vary over its lifetime. The efficiency factor enables this to be considered. Cost of water production analysis allows other quantitative methods to aid in the analysis and improvement of the cost of water services. The additional performance indicators help with further analysis and identifying areas requiring improvement. Table 1 introduces and interprets metrics that are relevant to the objective of this study.

The water production cost model is recommended for small scale community projects over conventional accounting methods for Capital projects such as net present value analysis. Sponsors, community leaders or researchers engaged with public health projects in small communities may not always have access to accountants. The water production cost model is a tool sufficient to help with basic analysis on the sustainability of the project. This model also does not consider the CAPEX as a bank loan which has to be repaid with interest over the life of the delivered project. The interest payment would be classified as expenditure if a bank loan was obtained to fund the project but is not applicable otherwise.

\section{Web based tool for model}

In order to allow the cost of water production tool to be easily applied by governments, NGOs and communities, a web tool has been developed. The tool will be able to provide analysis required when assessing new projects. The background calculator is an MS EXCEL ${ }^{\text {TM }}$ spreadsheet consisting of several calculation sheets with a single user interface sheet. The spreadsheet was converted to a web page file using Spreadsheet Converter Version 7.3.5. The web based tool comes with short instructions but requires users to be familiar with this paper. 
Table 1. Relevant metrics for water production cost evaluation.

\begin{tabular}{|c|c|c|}
\hline Component & Metric & Comments \\
\hline CAPEX & $\begin{array}{l}\text { CAPEX to expected Lifetime Water } \\
\text { Production Volume CAPEX: }(V \times t \times \sigma)\end{array}$ & $\begin{array}{l}\text { The CAPEX to expected water production volume } \\
\text { indicates the contribution capital cost makes to the } \\
\text { lifetime cost of water production. The capital cost } \\
\text { commences to exist as soon as the facility is built; } \\
\text { even before a single drop of water is produced. } \\
\text { Comparison can be carried out between design } \\
\text { options and alternate technologies. The same analysis } \\
\text { could be applied to individual components in the } \\
\text { system. }\end{array}$ \\
\hline $\begin{array}{l}\text { Operational } \\
\text { Expenditure }\end{array}$ & $\begin{array}{l}\text { Operational expenditure to expected Lifetime } \\
\text { Water Production Volume } O P E X \text { : } \\
\left(V_{\exp } \times t \times \sigma\right)\end{array}$ & $\begin{array}{l}\text { The operational expenditure to expected water } \\
\text { production volume indicates the contribution } \\
\text { operational cost has to the lifetime water production. } \\
\text { This cost is dependent on volume. High costs could } \\
\text { be impacted by the quality of feed as well as } \\
\text { operating strategy. }\end{array}$ \\
\hline Total Cost & Total cost of water production & $\begin{array}{l}\text { The total cost of water production can be used to } \\
\text { benchmark against other facilities, annual performance } \\
\text { and industry benchmarks. Analysis of the cost of } \\
\text { water production can be carried out using individual } \\
\text { sub-sections or using the CAPEX and OPEX to } \\
\text { lifetime water production volume metrics stated in this } \\
\text { table. }\end{array}$ \\
\hline
\end{tabular}

In the input fields, data about the service system to be designed is collected. As illustrated by Figure 2, the expected CAPEX, first year of operational expenditure and designed production volume is the input by the user. The expected values can be determined by using methodologies preferred by the user. Capital and operational expenditure are categorized in sub-elements to aid in data assembly. The operational expenditure sub-element fields are linked with fields in the lifecycle data section of the calculator.

In the lifecycle data section, variable inputs for up to five scenarios can be entered. The tool can be expanded to accommodate more scenarios if required. The three categories of input are system details, usage changes and price changes. In the system details, the user inputs the aging factor for the service, requirement for additional capital investment and expected service life. Plant aging helps compensate for performance loss of the system due to wear and tear of plant and other usage or aging related performance issues. The extra capital field allows users to state any additional capital investment that maybe required later in the life of the water service system. The expected plant life is an estimation of the life of the water service system before significant changes or reconstruction will be required.

The consumption and price increase fields require similar inputs. In both tables, sub-elements of the operational expenditure are stated. This allows individual component prices and forecast consumption increases to be input. The changes, input in percentages, are driven by the assumptions users make about the components.

The calculator uses all the input information and performs calculations for individual scenarios. The background spreadsheets allow calculation for every scenario. The calculation sheets are not accessible to the user. The output from the individual calculations is output to the main interface sheet. 

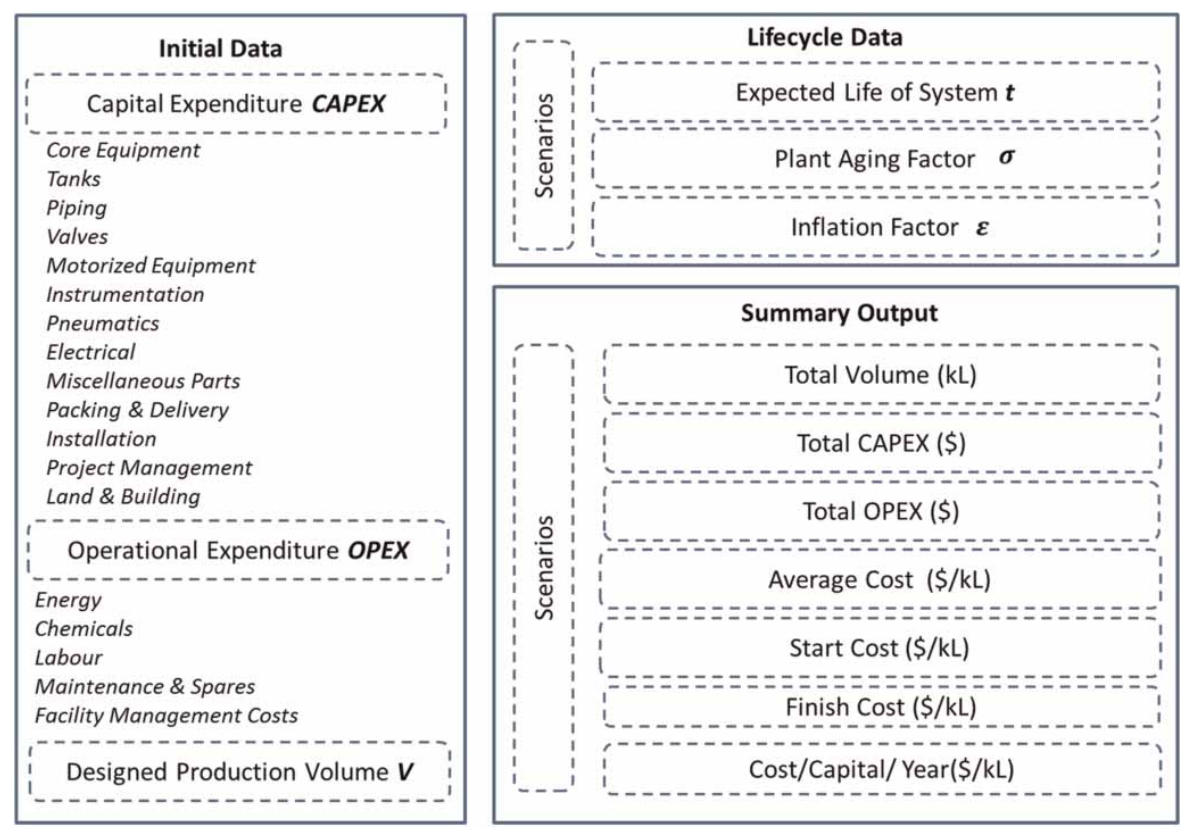

Fig. 2. Input data for the web-based water services lifecycle cost modelling tool.

In the web-based tool, detailed and summarized results are provided as output for each scenario. In the detailed output, the cost of water production is provided for each scenario by year. This allows detailed comparison of strategies on an annualized basis. The summary table, illustrated in the summary output section of Figure 2, provides a totalized comparison of the scenarios, useful for high-level reviews and making strategic decisions.

\section{Modelling}

For the purpose of this study, a theoretical water service system located in a small community was modelled. The 0.15 mega litres per day (MLD) system is intended to provide enough water to a community of 1,500 residents. The water service system was designed with an expected service life of 10 years. Table 2 provides a listing of components making up the initial CAPEX for the described water service system. For simplicity, cost of water production for the applicable components has been summed in Table 2.

The capital costs include systems that will treat feedwater and distribute it to users. The core equipment forms the bulk of the cost and assumes a stand-alone system capable of drawing raw feed water and pumping filtrate out for consumption. The tanks are for storage and are strategically sized to provide sufficient water for peak demand periods. Piping assures water is carried through closed channels safely from feed to the treatment set-up, and eventually to users. Motorized equipment represents additional equipment needed to supply feed and filtrate to the required locations. The system is designed with minimum instrumentation. The instrumentation ensures quality, safety and the reliability of the equipment. Electrical components include set-up of building, provision of electricity to the equipment, and 
Table 2. Categorization of initial CAPEX for a modelled water service system.

\begin{tabular}{lll}
\hline CAPEX & Costs & Totals (USD) \\
\hline Equipment & & $\$ 73,460$ \\
Core Equipment & $\$ 35,988$ & $\$ 10,212$ \\
Tanks & $\$ 6,432$ & $\$ 928$ \\
Piping & $\$ 5,388$ \\
Valves & $\$ 1,404$ \\
Motorized Equipment & $\$ 788$ \\
Instrumentation & $\$ 4,544$ \\
Pneumatics & $\$ 852$ & $\$ 924$ \\
Electrical & $\$ 6,000$ & \\
Misc Parts & & $\$ 10,000$ \\
Packing \& Delivery & & $\$ 10,000$ \\
Installation & & $\$ 93,460$ \\
Project Management & & \\
Land \& Building & & \\
Total CAPEX & & \\
\hline
\end{tabular}

lighting in the process and ancillary areas. There is a $\$ 10,000$ cost for project management, which has been significantly discounted when compared to commercial projects. This cost covers travel, verification and necessary regulatory approvals required for the system, building and other auxiliary establishments. The installation cost takes into consideration transportation of components, equipment and building supplies along with fitment and installation costs. The civil and building costs include costs associated with construction, preparation and erection of physical structure to house the necessary pipework and protection set-up required to protect the feedwater source.

Table 3 provides a break-down of the operational expenditure. The most critical and significant component is energy. The study assumes electricity is provided from existing sources in the location but if it is not, electricity generation sources need to be added to the CAPEX. Chemicals are required for treatment and sanitization. Chemicals are likely to be consumed for cleaning and washing of filters. It is assumed that filters, often membranes, will be used to filter the water. Operator labour costs have

Table 3. Categorization of initial OPEX of an example water service system.

\begin{tabular}{lll}
\hline Operating Expenditure (Year 1) & Costs & Totals \\
\hline Energy & & $\$ 1,248$ \\
Chemicals & & $\$ 292$ \\
Labour & & $\$ 730$ \\
Maintenance \& Spare Parts & $\$ 803$ & $\$ 1,003$ \\
Membranes & $\$ 200$ & \\
Other & $\$ 7$ & $\$ 58$ \\
Facility Management Costs & $\$ 29$ & \\
Raw Water & $\$ 22$ & $\$ 3,331$ \\
Water Disposal & & \\
Consumables & & \\
Total Operating Expenditure & & \\
\hline
\end{tabular}


been considered but this is dependent on the model adopted by the community where the service is offered or by the service provider. In a community-oriented set-up, the labour cost may be negligible. Maintenance costs consider consumables specifically for maintenance purposes and labour associated with the maintenance of the water service system.

Using the costing model detailed in the water production cost section, five scenarios were evaluated. These scenarios were hypothetically developed to provide an overview of the implications of various events or conditions on the overall cost of water production. The case studies will now be looked at in detail. Table 4 summarizes the possible operational scenarios studied.

Scenario 1. Scenario 1 is the ideal case scenario applicable where the water service system performs without any issues for its entire lifetime. It assumes a plant-aging factor that sees a $2 \%$ decline in filtrate output annually. Scenario 1 also assumes a 1 to $4 \%$ annual inflation of operational cost components.

Scenario 2. Scenario 2 considers the case of a difficult feed, which is the likely case for small locations where groundwater might be used. Groundwater will be laden with minerals or have heavy organics loading making treatment difficult. In modelling this scenario, minor increases in energy, filters and operating labour were considered. Considering the feed, there will be excessive fouling of the filters. Hence, significant increases in chemical consumption and consumables were assumed. The model, similar to Scenario 1, assumes inflation of operational costs.

Scenario 3. Scenario 3 leads on from Taylor (2009) where it was found that equipment often implemented for WASH services in remote areas could be unreliable. This could be attributed to inadequate system design or implementation of a system not robust enough to sustain operational challenges in the field. To ensure a 10-year lifetime performance, the model assumes an injection of additional capital on top of increases in operational expenditure. Significant increases are assumed for chemical consumption and other consumables along with minor increases noted for energy, filters and operating labour. Scenario 3 could also be applicable in events where capital is required to reconstruct the water service after a natural disaster like flood, cyclone or earthquake.

Scenario 4. Upfront costs can sometimes be difficult to fund and communities could opt for lower capital solutions with the intention to operate the water service system with higher operating costs. This could be accomplished by not using pre-treatment options, using cheaper components and as a result opting for more frequent maintenance activities. For this scenario, a significantly lower capital

Table 4. Brief description of simulation scenarios.

\begin{tabular}{lllll}
\hline \multirow{2}{*}{ Scenario } & Scenario Summary & Plant Aging & Operational Exp Change OPEX, & Capital Exp Change \\
& $\sigma$ & $\varepsilon$ & CAPEX \\
\hline 1 & Normal & Normal & No Change & Normal \\
2 & Difficult Feed Water & High & Minor Increase & Normal \\
3 & Unreliable Equipment & Normal & Minor Increase & Normal \\
4 & Low Capital, High Operational & Normal & Major Increase & Low \\
& Exp. & Nil & Minor Increase & Normal \\
\hline
\end{tabular}


cost was taken alongside major increases to operational expenditure. In the operational expenditure, minor increases were provided for energy, filters and operating labour while factoring significant increases in the consumption of chemicals, consumables and maintenance.

Scenario 5. Scenario 5 is similar to Scenario 3 and leads on from Taylor (2009) where reference is made to non-functional systems scattered in small communities globally. These scenarios are not planned for and could be a result of poor design, natural and manmade disasters or simply unreliable equipment that cannot be repaired. The model assumes that no effort is made to repair the equipment and hence the operational expenditure remains comparable to typical values.

The five scenarios presented above capture real life events experienced by water service systems. From deterioration through normal usage or destruction through calamities, the scenarios summarize challenges water service system designers and implementers need to be aware of.

\section{Results}

Data presented in Tables 2, 4 and 5 were used to calculate the cost of water production. Equation (3) was used for the determining the eventual cost of water production. Figure 3 represents the output of the tool for each of the scenarios discussed earlier.

Table 5 elaborates on Figure 3 and then summarizes the water production costs for each of the scenarios.

Table 5. Cost of water production for simulated scenarios.

\begin{tabular}{llll}
\hline \multirow{2}{*}{ Scenario No. } & \multicolumn{2}{l}{ Cost of Water Production $\left(\$ / \mathrm{m}^{3}\right)$} & Year 10 \\
\cline { 2 - 4 } & Mean & Year 1 & $\$ 0.28$ \\
1 & $\$ 0.26$ & $\$ 0.23$ & $\$ 0.54$ \\
2 & $\$ 0.34$ & $\$ 0.23$ & $\$ 0.32$ \\
3 & $\$ 0.28$ & $\$ 0.25$ & $\$ 0.28$ \\
5 & $\$ 0.24$ & $\$ 0.20$ & - \\
\hline
\end{tabular}

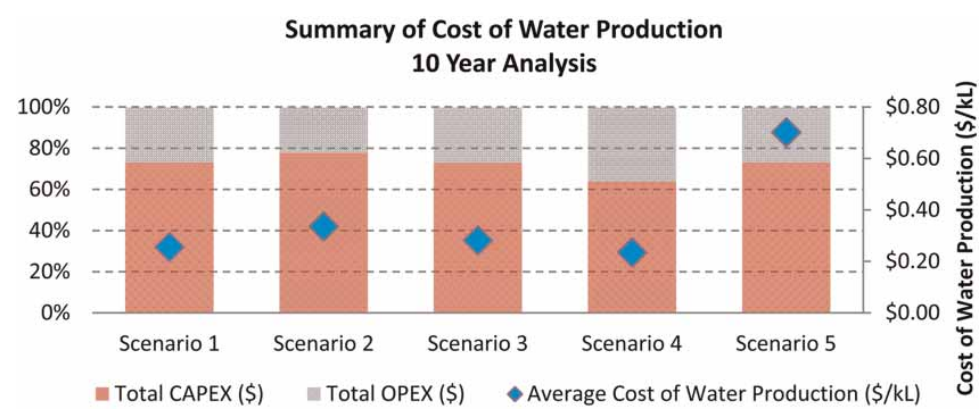

Fig. 3. Summary of the analytical estimation tool for simulation scenarios. 


\section{Discussion}

Analysis of the cost of water production and the average cost of water production is provided hereafter.

\section{Scenario 1}

Most community projects would like to operate this way. This scenario is therefore the base scenario. Performance shift is due to natural aging of equipment. There is a $17 \%$ reduction in volume of water produced in year 10 , relative to year 1 with a $2 \%$ increase in cost. This resulted in a $22 \%$ shift in the cost of water production over the 10 year period.

\section{Scenario 2}

As stated earlier, this is the scenario with difficult groundwater, laden with minerals. Water production is less and is a result of excessive fouling which warrants increased frequency of cleaning and maintenance. Without the cleaning and maintenance, water production volumes and the lifecycle equipment performance will be severely compromised. In other words, regular cleaning and maintenance cycles aid in bringing the equipment performance comparable to Scenario 1. The maintenance and cleaning regime causes a $61 \%$ decrease in overall volume. Due to less volume production, the operating cost is lower. This is despite the increased operational expenditure due to chemical consumption. There is $29 \%$ increase in the cost of water production over the 10 year period. Additional capital investment can assist to lower the costs and/or increase the output if the volume of water produced is less than the demand. A pre-treatment step could be added to the set-up.

\section{Scenario 3}

Unexpected disasters that damage equipment or inadequate design and planning that result in unreliable performance of equipment drive this scenario. Capital is invested to sustain performance of the system. The model had assumed an injection of $10 \%$ additional capital at start-up. While the volume is comparable to Scenario 1, the cost of water production is marginally higher. Despite the cost of water production being nearly comparable, this scenario indicates that communities need to keep funds to repair and reinstate services if needed. If additional capital funds are not available, severe disruptions to water services will be caused and established equipment will be abandoned with no long term benefit.

\section{Scenario 4}

Lower upfront capital costs can often allow communities to afford small-scale water treatment projects. In this model, the lower capital cost was translated to increased operational expenditure. The output of the model indicated that the volume of water production was comparable to Scenario 1 and so was the cost of water production after 10 years. This could be a result of the assumptions of the model; the decrease in CAPEX was given the same weighting as the increase in operational expenditure. This may not hold true for all applications and possibly warrant analysis by designers and planners early 
in the design and decision phase of projects. For example if inflation rates were higher or additional capital were required after a few years, the cost of water production may differ from Scenario 1.

\section{Scenario 5}

The failed equipment scenario extends from Scenario 3 where due to disasters or inadequate design, water production equipment ceases to function. While in Scenario 3, through capital injection the equipment function is restored, Scenario 5 is based on the equipment only having 3 years of life. This translates to a $100 \%$ decline in water production volume after the expected 10 year life and significantly increases the cost of water production. There are no savings, and investment in a system that cannot produce water through its expected life ends up being a significant economic loss. If only a 3 year life was taken, the cost of water production, given the capital investment, would still be considerably high. Based on this scenario, the recommendation that additional capital funds must always be available, suggested in Scenario 3, is reaffirmed. This is very likely to happen in situations where long-term risks have not been thoroughly considered and contingency arrangements made.

To summarize, the water services facility, based on the evaluation presented, should endeavour to keep its capital and operational expenditure low with a reliable high volume output. While this is stating the obvious, high water production volume has a favourable influence on the cost of water. Hence, it is important that the equipment not only reliably produces water but it is also sized to achieve a reasonable cost of water. An oversized unit will keep capital and operational costs beyond affordability. Furthermore, the water services facility, in order to be economically sustainable, should always remain in service. Hence, the system and supporting infrastructure needs to be durable and robust to withstand calamities. This means that preventative maintenance systems or maintenance plans recommended by suppliers will need to be implemented to minimize operational costs and reduce the likelihood of significant unplanned expenditure.

\section{Conclusions}

An analytical tool for modelling water service systems for small community projects was developed and demonstrated. The model considered the components illustrated in Figure 1, such as the CAPEX necessary for the establishment of the service system and operational expenditure required for functioning of the water service system.

In this study, it was shown that the tool could be used to evaluate the performance of a water service system. Using five test scenarios, the analysis showed that capital and operational costs should be low while ensuring high volumes of water production. In addition to achieving low costs and high fluxes, water service systems of the future need to achieve increased lifetime; by withstanding challenges faced in small communities.

The tool therefore enables effective decision making for governments, non-governmental agencies and project sponsors by testing alternatives and modelling potential events in the water service system's lifecycle to assess economic impact. The tool will also aid researchers, technology developers and research funding agencies in comparing and accelerating the development of new technologies that will further lower the cost of water production and improve public health as a result. 


\section{References}

Bartram, J. (2008). Improving on haves and have-nots. Nature 452(7185), 283-284.

Brownell, S. A., Chakrabarti, A. R., Kaser, F. M., Connelly, L. G., Peletz, R. L., Reygadas, F., Lang, M. J., Kammen, D. M. \& Nelson, K. L. (2008). Assessment of a low cost, point of use, ultraviolet water disinfection technology. Journal of Water and Health 6(1), 53-65.

Clasen, T., Haller, L., Walker, D., Bartram, J. \& Cairncross, S. (2007). Cost-effectiveness of water quality interventions for preventing diarrhoeal disease in developing countries. Journal of Water and Health 5(4), 599-608.

Cronin, A., Shrestha, D., Cornia, N., Abdalla, F., Ezard, N. \& Aramburu, C. (2008). A review of water and sanitation provision in refugee camps in association with selected health and nutrition indicators - the need for integrated service provision. Journal of Water and Health 6(1), 1-13.

Graham, J. P. \& VanDerslice, J. (2007). The effectiveness of large household water storage tanks for protecting the quality of drinking water. Journal of Water and Health 5(2), 307-313.

Haller, L., Hutton, G. \& Bartram, J. (2007). Estimating the costs and health benefits of water and sanitation improvements at global level. Journal of Water and Health 5(4), 467-480.

Hutton, G., Haller, L. \& Bartram, J. (2007). Global cost-benefit analysis of water supply and sanitation interventions. Journal of Water and Health 5(4), 481-502.

Jacangelo, J. G., Askenaizer, D. J. \& Schwab, K. (2006). Research needs in drinking water: a basis in regulations in the United States. Journal of Water and Health 4(Supplement), 1-9.

Kayser, G. L., Moriarty, P., Fonseca, C. \& Bartram, J. (2013). Domestic water service delivery indicators and frameworks for monitoring, evaluation, policy and planning: a review. International Journal of Environmental Research and Public Health 10, 4812-4835.

Kumar, S., Groth, A. \& Vlacic, L. (2014). An analytical index for evaluating manufacturing cost and performance of lowpressure hollow fibre membrane systems. Desalination 332(1), 44-51.

Moe, C. L. \& Rheingans, R. D. (2006). Global challenges in water, sanitation and health. Journal of Water and Health 4(Supplement), 41-57.

Muhammad, N., Sinha, R., Krishnan, R., Patterson, C., Haught, R. C., Harms, H. \& Seville, R. (2010). Evaluating a composite cartridge for small system drinking water treatment. Journal of Water and Health 8(2), 212-223.

Murphy, H., McBean, E. \& Farahbakhsh, K. (2010). A critical evaluation of two point-of-use water treatment technologies: can they provide water that meets WHO drinking water guidelines? Journal of Water and Health 8(4), 611-630.

Rizak, S. \& Hrudey, S. (2008). Drinking - water safety - challenges for community managed systems. Journal of Water and Health 6(S1), 33-41.

Sullivan, C. A., Meigh, J. R. \& Giacomello, A. M. (2003). The water poverty index: development and application at the community scale. Natural Resources Forum 27(3), 189-199.

Taylor, B. (2009). Addressing the Sustainability Crisis - Lessons from Research on Managing Rural Water Projects. Water Aid, Dar es Salaam.

Received 16 June 2015; accepted in revised form 9 November 2015. Available online 22 December 2015 